\title{
TEMA 1 -2018: Actualidad en el papel de la Cladribina en el tratamiento de la Esclerosis Múltiple tipo brote-remisión
}
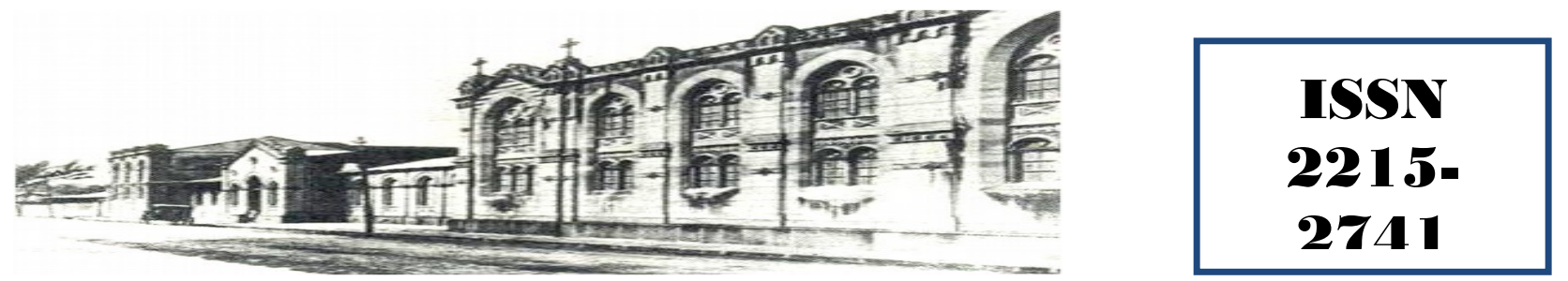

Haspital San quan de Dias, San Jasé, Casta Rica. Fundada en 1845

Recibido: $\quad 06 / 12 / 2017$

Aceptado: $\quad$ 15/01/2018

${ }^{1}$ Medico Cirujano General. Graduado de la Universidad de Costa Rica. carlossq24@hotmail.com

Carlos Mario Sequeira Quesada ${ }^{1}$ José David Villegas Reyes ${ }^{2}$

${ }^{2}$ Medico Residente en la Especialidad de Neurología. Hospital México y Hospital Calderón Guardia.

\section{RESUMEN}

La esclerosis múltiple es una enfermedad crónica inflamatoria de naturaleza autoinmune caracterizada por compromiso del sistema nervioso central mostrando afectación de la sustancia blanca y neurodegeneración, la cual se puede clasificar en primariamente progresiva, secundariamente progresiva y forma brote-remisión. En la actualidad la Food and Drugs Administration (FDA) ha aprobado un total de 11 drogas para el tratamiento de la esclerosis múltiple en su variante broteremisión. La terapéutica de esta entidad en Costa Rica está dictada principalmente por la Guía Nacional para el Tratamiento de la Esclerosis Múltiple, la cual incluye opciones de primera y segunda línea tanto orales como parenterales. A nivel mundial, las principales investigaciones y avances farmacológicos referentes a la esclerosis múltiple se han dirigido al desarrollo de opciones terapéuticas orales. Dentro de la gama de opciones estudiada está la cladribina, análogo sintético de las purinas, el cual a pesar de resultados positivos en los estudios llevados a cabo fue aislada de intentos de aprobación debido a preocupaciones sobre posible causalidad con infecciones y neoplasias inherentes a su mecanismo de acción. Investigaciones recientes sin embargo han cuestionado estas preocupaciones y el fármaco se mueve hacia una eventual aprobación a nivel europeo e incorporación en las guías terapéuticas de la esclerosis múltiple forma brote-remisión.

\section{PALABRAS CLAVE}

Esclerosis Múltiple, Brote-Remisión, Cladribina, cáncer

\section{ABSTRACT}

Multiple sclerosis is a chronic inflammatory disease of autoimmune nature characterized by compromise of the central nervous system white substance and neurodegeneration. Multiple sclerosis can be catalogued as primary progressive, secondary progressive and relapseremission. At the moment, the Food and Drugs Administration (FDA) has approved a total of 11 
drugs for the therapeutic approach of relapseremission multiple sclerosis. In Costa Rica treatment for this entity is mainly dictated by the National Guideline of Treatment for Multiple Sclerosis, which includes oral and parenteral options in both first and second line of treatment. Around the world, research and pharmacological breakthroughs pertaining multiple sclerosis have been geared towards developing oral options. Among the variety of options studied for this goal is cladribine, a synthetic purine analogue, which even though presented positive results in its clinical trials, was removed from consideration for an approval license due to concerns related to a possible causality link with infections and cancer, intrinsic to the action mechanism of the cladribine. Recent clinical trials nonetheless have questioned this doubts and cladribine is gearing towards approval in Europe and incorporation in the respective guidelines for the treatment of relapse-remission multiple sclerosis.

\section{KEY WORDS}

Multiple Sclerosis, Relapse-Remission, Cladribine, cancer.

\section{INTRODUCCIÓN}

La esclerosis múltiple es una enfermedad autoinmune mayor, que afecta el sistema nervioso central (SNC), con una etiología compleja y multifactorial incluyendo interacciones entre aspectos genéticos del individuo y el medio ambiente que le rodea. En la fisiopatología de esta entidad se citan como factores agentes infecciosos, tabaquismo, bajos niveles de vitamina $\mathrm{D}$ así como la ubicación latitudinal del individuo. La esclerosis múltiple es caracterizada por lesiones desmielinizantes diseminadas en tiempo y espacio, comprometiendo principalmente la sustancia blanca, en ausencia de una mejor explicación para el cuadro clínico, asociando además gliosis y pérdida neuronal. La misma naturaleza multifocal de esta entidad desmielinizante explica lo variado que puede ser su cuadro clínico, y lo diversas que son las manifestaciones de la mismas, las cuales varían entre lo abrupto y lo insidioso, lo trivial y lo discapacitante. A pesar de lo anterior, las lesiones desmielinizantes presentan una predilección por ciertas partes del sistema nervioso central, permitiendo reconocer como distintivas de esclerosis múltiple a determinados complejos de síntomas, signos y patrones en los estudios de imágenes. Los síntomas y hallazgos físicos de esta entidad incluyen neuritis óptica, afectación de las vías oculomotoras y otros pares craneales, alteraciones sensitivas, manifestaciones motoras, ataxia cerebelosa, compromiso de los esfínteres y las funciones sexuales, fatiga, compromiso a nivel cognitivo y desórdenes afectivos. ${ }^{1234}$.

La historia clínica y la exploración física buscando y documentando todas las manifestaciones previamente citadas se consideran el pilar diagnóstico junto a estudios de imágenes como la resonancia magnética. En este aspecto se utilizan principalmente los criterios diagnósticos de McDonald, con su última revisión en el año 2010. Además, se recurre en la práctica diaria a valiosa información paraclínica complementaria brindada por la punción lumbar, la realización de potenciales evocados visuales y la tomografía de coherencia óptica ${ }^{12}$.

El tratamiento farmacológico para esta patología ha venido ampliándose en las últimas dos décadas. En la actualidad se ha hecho rutina que los neurólogos clasifiquen a cada paciente según la forma clínica de la enfermedad, para efectuar las intervenciones terapéuticas en el momento oportuno, reconocer los casos con falla terapéutica o la presencia de eventos adversos inaceptables ${ }^{1}$. Alrededor del $85 \%$ de los casos de esclerosis múltiple tienen una presentación inicial en forma de brotes con remisión, en donde se presentan eventos clínicos de focalización neurológica, con recuperación parcial o total y recurrencia en otro momento, en el mismo sitio neuroanatómico o en otro diferente. Con el paso del tiempo, en la mayoría de los pacientes los brotes van dejando gliosis en el SNC por lo que se va acumulando la discapacidad, reemplazándose la aparición de brotes por un cuadro caracterizado principalmente por degeneración y atrofia. Este paciente se reconoce como con una forma clínica secundaria progresiva. El restante $10-15 \%$ de los casos pueden presentarse como una forma progresiva de deterioro neurológico por lesiones 
desmielinizantes, sin francos eventos singulares desmielinizantes, a esta forma se le conoce como esclerosis múltiple primaria progresiva ${ }^{134}$.

El tratamiento de la esclerosis múltiple ha tenido una revolución importante en las últimas dos décadas, comenzando con la introducción del interferón $\mathrm{B}$ como primera droga dirigida para modificar el curso natural de esta enfermedad, y dando inicio a un desarrollo subsecuente de nuevas opciones terapéuticas, siendo este un campo que ha progresado rápidamente, brindando a los galenos y pacientes una amplia gama de opciones para el manejo de la enfermedad. En la actualidad un total de 11 fármacos tienen aprobación de parte de la FDA. La mayoría de esquemas terapéuticos considerados clásicos para la esclerosis múltiple centran mayoritariamente su primera línea de abordaje en fármacos de aplicación inyectable, como el interferón beta-1a y b, así como el acetato de glatiramer. Estas mismas drogas están ampliamente disponibles a nivel mundial, sin embargo, persiste una preocupación sobre la adherencia terapéutica y la eficacia de las mismas, además es dudoso en este momento si estas drogas tienen eficacia deteniendo o enlenteciendo la neurodegeneración, aspecto de la esclerosis múltiple cada vez más reconocido. Debido a estas dudas, el enfoque en el campo terapéutico de esta enfermedad desmielinizante se centró sobre el desarrollo y aprobación de drogas de administración oral, las cuales promueven satisfacción del paciente y aumentan el cumplimento del tratamiento, manteniendo o superando evidentemente los resultados de las opciones terapéuticas clásicas. Entre estos recursos se cita al fingolimod, mitoxantrona, teriflunamida y dimetilfumarato como fármacos con aprobación por diferentes entes reguladoras mundiales. Otro recurso terapéutico utilizado son los anticuerpos monoclonales, grupo de donde se desprenden fármacos como el natalizumab, alentuzumab y el ocrelizumab 12356789

Numerosos ensayos clínicos se encuentran en curso, evaluando moléculas dirigidas contra el marcador de superficie CD20 y el receptor de la interleuquina (IL) 2, antagonistas selectivos del receptor la esfingosina-1-fosfato, el estriol y agentes promovedores de la remielinización. Además, agentes como la azatioprina, metotrexate, ciclofosfamida e inmunoglobulina intravenosa son usados en diversos centros de manera off-label. Se cuenta empero con otras drogas de ingestión oral con resultados prometedores, esperando la aprobación correspondiente de entes reguladoras, su incorporación a las guías de tratamiento mundiales o la finalización de sus respectivos ensayos clínicos. Uno de estos fármacos es la cladribina, agente quimioterapéutico inicialmente aprobado como tratamiento de la leucemia de células vellosas y que en fechas recientes ha vuelto a la palestra como la novedad terapéutica en el abordaje de la esclerosis múltiple tipo brote y remisión luego de haber sido dejado de lado por muchos años, debido a una controversia referente a sus efectos adversos en el año 2011. A lo largo de este trabajo se revisará la evidencia científica disponible para este fármaco y se establecerá su sitio actual en los esquemas de tratamiento y la postura de la comunidad médica respecto a su uso ${ }^{38}$.

\section{DISCUSIÓN}

\section{Esclerosis Múltiple en Costa Rica}

De acuerdo con la historia moderna, con el ahorro estatal obtenido por la abolición del ejército nacional en 1948 se pudo seguir impulsando la seguridad social a través de la Caja Costarricense del Seguro Social (CCSS), institución que había nacido siete años antes. Este ente cubre la totalidad de los estudios diagnósticos y los tratamientos de los pacientes con esclerosis múltiple del país. La población costarricense es actualmente de alrededor de 5 millones de personas, de las cuales aproximadamente un $95 \%$ tienen ascendencia genética predominantemente de origen europeo mediterráneo, con una prevalencia calculada de Esclerosis Múltiple de alrededor de 8 casos por 100000 habitantes. Esto se debe contextualizar contra una prevalencia de 1 por 100000 en áreas ecuatoriales, 6-14 por 100000 en el sureste de los Estados Unidos, 30-80 por 100000 en Canadá y el norte europeo y de los Estados Unidos ${ }^{14}$.

Para el año 1991 se publicaron los primeros dos casos de esta patología a nivel local. Estos pacientes fueron estudiados con potenciales evocados, y los autores de esa revisión concluyeron que los potenciales evocados podían 
constituirse en el principal examen complementario en el diagnóstico de esclerosis múltiple en el medio costarricense de ese momento, carentes entonces de resonancias y exámenes electroforéticos confiables. La primera resonancia magnética en nuestro país fue introducida en 1994, lográndose que 15 años después la CCSS adquiriera también sus dos primeros equipos resonadores. Actualmente en la práctica clínica neurológica todos los pacientes en los cuales se sospecha esta patología desmielinizante, son estudiados de una forma integral y de acuerdo a los criterios de McDonald previamente comentados. La CCSS ha logrado mejorar considerablemente entonces en el abordaje, detección y tratamiento de esta enfermedad. En el 2004 se tenían bajo tratamiento a 52 pacientes y al cierre del 2012 a 264. Según datos de la Asociación Costarricense de Esclerosis Múltiple para el 2015 se estimaba que en promedio en Costa Rica existían 460 casos detectados de esta patología, y Costa Rica es uno de los pocos países de América donde los pacientes con esta enfermedad crónica reciben cobertura por parte de la seguridad social ${ }^{11011}$.

Dentro de los lineamientos actuales de tratamiento de la esclerosis múltiple, los que más han moldeado la práctica en el medio nacional son las guías canadienses, algoritmos de manejo en España y el consenso latinoamericano. Utilizando este conocimiento como base, así como la experiencia acumulada a nivel local, surgió una guía Nacional para el Tratamiento de la Esclerosis Múltiple desarrollada por la Asociación Costarricense de Ciencias Neurológicas. La guía no impone criterios antojadizos, sino que ofrece una revisión de la evidencia actual del manejo inmunomodulador, adaptando literatura y guías internacionales, a las condiciones y posibilidades de los centros hospitalarios a nivel nacional. A falta de un documento institucional oficial por parte de la Caja Costarricense del Seguro Social, esta guía se erige como el único trabajo autóctono para basar el tratamiento de la Esclerosis Múltiple en Costa Rica ${ }^{1}$.

En la guía se establecen recomendaciones para ataques de brotes agudos, síndromes clínicos aislados y síndromes radiológicos aislados, así como comentarios sobre la evidencia en ese momento disponible para abordar las formas progresivas de la enfermedad, sin embargo, en esta revisión únicamente se hará referencia a los apartados de la Guía Nacional para el Tratamiento de la Esclerosis Múltiple referentes a la forma brote-remisión de esta patología ${ }^{1}$.

En el documento se enfatiza lo amplio del manejo de la esclerosis múltiple, citando la existencia de medidas no farmacológicas como terapia física, ocupacional y psicoterapia, los tratamientos sintomáticos como los analgésicos, antidepresivos y tratamiento para la espasticidad o problemas con vejiga neurogénica para citar algunas de las comorbilidades frecuentes, así como los medicamentos inmunomoduladores de la enfermedad. Esta última es separada en fármacos de primera línea y drogas de segunda línea. Cada línea de tratamiento es a su vez subdividida según su manera de administración, teniéndose fármacos orales así como fármacos parenterales, pudiendo ser estos últimos intravenosos, subcutáneos o intramusculares (Ver imagen \#1) ${ }^{1}$.

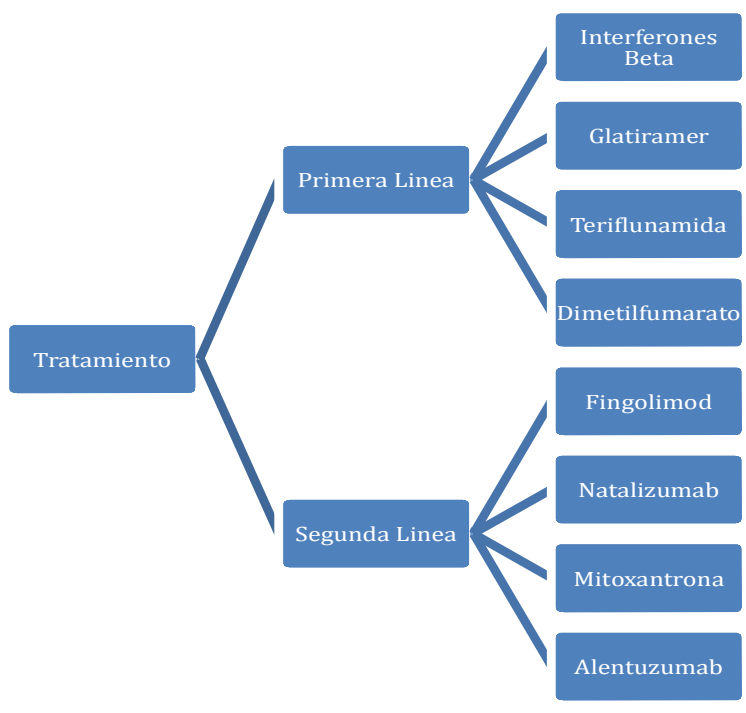

Fig. 1: Fármacos disponibles en Costa Rica para el abordaje de la esclerosis múltiple tipo brote-remisión, clasificados según primera y segunda línea de utilización.

Fuente: Guía Nacional para el Tratamiento de la Esclerosis Múltiple. 2015

La lógica para la escogencia del primer medicamento a utilizar en el tratamiento de la esclerosis múltiple brote y remisión sigue las recomendaciones internacionales y latinoamericanas, siendo 
las mismas adaptadas a las opciones terapéuticas presentes en el país. Si un paciente presenta una discapacidad menor objetivizada con escalas como la Expanded Disability Status Scale (EDSS), una baja carga lesional en la resonancia magnética, significando esto menos de 9 lesiones hiperintensas en secuencia T2/FLAIR, mientras que menos de 2 lesiones que refuercen con gadolinio en $\mathrm{T} 1$ indican una actividad inflamatoria menor, se le deberá iniciar tratamiento con interferón beta-1a intramuscular. Si por lo contrario un paciente con las mismas características, a excepción de una mayor carga lesional en la resonancia magnética, se le deberá dar tratamiento con interferón beta-1b. Un paciente que además de la mayor carga lesional tenga discapacidad media según la escala de EDSS, deberá instaurarse terapéutica con interferón beta-1a subcutáneo. Cualquier paciente cuyo puntaje en la escala de EDSS sea concordante con una discapacidad mayor se les deberá empezar tratamiento con alguna de las opciones citadas como de segunda línea. Esta selección debe ser guiada mediante la determinación de serología para el virus JC, así como el previo uso o no de algún otro inmunosupresor 1 .

En un apartado titulado como otras opciones terapéuticas con evidencia controversial, la cladribina es mencionada en esta guía nacional para el tratamiento de la esclerosis múltiple. Es citada la evidencia que respalda su efectividad en cuanto a la reducción de carga lesional y el tiempo para la presentación de la primera recaída, la cual será ampliada en esta misma revisión posteriormente, sin embargo, concluye mencionando la controversia referente a sus efectos adversos y supuesta débil evidencia que llevó en 2011 al retiro de la forma comercial del medicamento oral. La guía, por motivos de fecha de publicación, no hace mención a la evidencia que ha devuelto a este medicamento a un punto de discusión como opción terapéutica, misma que también será analizada en este documento ${ }^{1}$.

\section{Características Farmacológicas de la Cladribina}

Las drogas inmunosupresores son de importancia terapéutica significativa en el tratamiento de múltiples enfermedades autoinmunes. La cladribina, o 2-chlorodeoxyadenosa, es un nucleósido tipo purina resistente a la deaminasa de adenosi- na, fungiendo justamente como droga de índole inmunomodulador con aprobación para el tratamiento de la leucemia de células vellosas y con conocida efectividad en la esclerosis múltiple. Este fármaco presenta un efecto apoptótico principalmente pero no limitado a linfocitos, el cual no es dependiente de la proliferación celular ${ }^{812}$ 13 .

El mecanismo de acción de este quimioterapéutico, a pesar de no encontrarse completamente dilucidado en el contexto de la esclerosis múltiple, en rasgos generales consiste en el ingreso de la molécula a la célula correspondiente a través de los transportadores de purina, y una vez en el interior de la misma la cladribina es fosforilada por la deoxicitidina quinasa, la cual es la enzima limitante en el proceso de activación de la cladribina. En las células en las cuales la relación entre deoxicitina quinasa y deoxinucleotidasa es alto, como lo es los linfocitos, la cladribina es fosforilada a la forma activa, llamada 2-chlorodeoxyadenosina-ATP ${ }^{8}$.

Este deoxinucleotidotrifosfatado se acumula llevando a disrupción del metabolismo celular y lleva a daño en $\mathrm{ADN}$, lo cual conlleva muerte celular. Esta apoptosis es inducida por la activación de diferentes caspasas como la 3, 8 y 9. Este proceso eventualmente desencadenará en depleción linfocitaria y una linfopenia persistente ${ }^{8}$.

El mecanismo anterior está más claramente dilucidado y validado en los linfocitos, como se mencionó al inicio. Sin embargo, los efectos de la cladribina en otras células del sistema inmune no son completamente comprendidos a pesar de saberse existentes. La cladribina también actúa en células dendríticas, tanto maduras como inmaduras derivadas de monocitos, y monocitos humanos. Tratamiento con este fármaco indujo apoptosis luego de 24 horas en los monocitos, mientras que este efecto fue observado en las células dendríticas 72 horas después. El tratamiento con cladribina causó inducción en monocitos de las caspasas 3 y 9, no así en células dendríticas. Referente a efectos a nivel mitocondrial, se observó que el potencial de membrana se encontraba fuertemente reducido en las células dendríticas más no en monocitos. Los hallazgos anteriores denotan que la cinética en el metabolismo de la cladribina es diferente entre células dendríticas, monocitos y linfocitos ${ }^{12}$. 
Aparte de la inducción de apoptosis de los diferentes agentes del sistema inmune se han discutido otros efectos de la cladribina como la reducción de niveles en líquido cefalorraquídeo de interleuquina 8, así como apoptosis de microglia a nivel de sistema nervioso central. Otros estudios han encontrado un aumento en la cantidad circulante de interferón alfa durante el tratamiento. Estos hallazgos, así como las conclusiones de otros estudios, demuestran que la cladribina puede tener diferentes mecanismos de acción independientes de la inducción de muerte linfocitaria 121415

Se debe resaltar además el rol de la proteína p53. Es bien sabido que es una proteína clave que se activa en respuesta al estrés celular. Estudios demuestran que tanto los monocitos como células dendríticas tiene activación de la citada proteína, sin embargo, la cinética de esta activación es retrasada en las células dendríticas en comparación con los monocitos. Esta diferencia y el rol del p53 en las redes apoptóticas principales podría implicar que el momento de activación de esta proteína regule precisamente las cinéticas de apoptosis ${ }^{12}$.

Resulta además importante establecer específicamente en cual población de linfocitos ejerce preferencialmente la cladribina sus efectos, siendo esto clave no solo para comprender las diferencias en efectividad con otros medicamentos utilizados en los esquemas de tratamiento, sino también por los diferentes perfiles de seguridad y ocurrencia de efectos adversos que se obtienen con la cladribina y demás fármacos. A pesar de que, como se ha dicho, el mecanismo de acción en la esclerosis múltiple del quimioterapéutico en cuestión no está completamente dilucidado, y en la misma fisiopatogenia de la entidad desmielinizante no se ha dicho la última palabra, la eficacia de ciertos medicamentos como el alentuzumab se ha atribuido a deleción de linfocitos tipo T CD4 con una conservación de células $\mathrm{T}$ reguladoras. Sin embargo, otros fármacos que se han demostrado efectivos como el ocrelizumab basan su posible mecanismo más bien en poblaciones de linfocitos tipo $\mathrm{B}^{516}$.

Estudios han demostrado que la cladribina muestra un perfil de acción consistiendo en causar una depleción modesta en linfocitos $\mathrm{T}$ y $\mathrm{NK}$, pero una eliminación todavía más fuerte de células tipo B. Esta depleción poblacional tarde en promedio 1 a 3 meses si se utiliza las dosis máximas del fármaco. Se observa además que la cladibrina causa una marcada y duradera depleción preferencial de células tipo $\mathrm{B}$ con el marcador de superficie CD19, las cuales luego de un año de tratamiento aún no habían vuelto a niveles basales. En la actualidad no existe información objetiva sobre influencia de la cladibrina en células B de memoria, sin embargo la efectividad de esta droga suprimiendo la actividad en la esclerosis múltiple podría indicar efectivamente acción del fármaco sobre estas poblaciones ${ }^{16}$.

Para referirse a la farmacocinética de la cladribina se debe restablecer que se tienen formulaciones parenterales, dirigidas principalmente a la terapia de la leucemia, así como presentación oral, la cual ha sido precisamente la explotable en el abordaje para la esclerosis múltiple. Un estudio realizado por Savic et al encontró que la farmacocinética de la cladribina se podría describir siguiendo un modelo de tres compartimientos, mientras que la de su metabolito, la 2-chloroadenina, como un modelo de un único compartimiento. El aclaramiento renal del primero fue correlacionado con el aclaramiento de creatinina, prediciendo un aclaramiento de 19\%, 30\% y 40\% para pacientes con aclaramientos de creatina de 65,40 y $20 \mathrm{ml} / \mathrm{min}$ respectivamente. Los alimentos tuvieron un efecto de disminución sobre la absorción de un $11.2 \%$, además de causar un retardo en la misma ${ }^{17}$.

\section{Evidencia Científica para el Uso de la Cladribina}

Estudios clínicos comprobando la eficacia este fármaco como recurso en el tratamiento de la esclerosis múltiple inician remontándose a publicaciones tan temprano como en 1994 y 1996, cuando dos estudios fase II involucrando pacientes con esclerosis múltiple tipo progresiva recibiendo $0.7 \mathrm{mg} / \mathrm{kg}$ de cladribina se asociaron con una estabilización significativa de la enfermedad, siendo esta objetivizada con escalas como la EDSS y SNRS. Estos resultados iniciales motivaron la evaluación de esta molécula en estudios multicentro para el abordaje de la esclerosis múltiple progresiva, como el llevado a cabo por el Cladribine MRI Study Group, sin embargo, no se observaron cambios significativos en las escalas 
previamente mencionadas al emprender este tratamiento $^{818}$.

Es sin embargo en el abordaje de la esclerosis múltiple en su variante tipo brote remisión donde se ha centrado la mayoría de estudios de la cladribina y a su vez donde los resultados han sido más impresionantes. No han sido los únicos estudios llevados a cabo, sin embargo se debe resaltar los resultados obtenidos por los ensayos clínicos CLARITY y ORACLE MS, los cuales constituyen la evidencia científica más relevante sobre el rol del medicamento mencionado en esta patología desmielinizante ${ }^{8}$.

El estudio ORACLE MS fue un ensayo fase III randomizado, multicéntrico, doble ciego donde se investigaron los efectos de la cladribina oral en la conversión a esclerosis múltiple clínicamente definitiva en pacientes con un primer evento clínico de desmielinización. El estudio fue llevado a cabo con 903 pacientes con edades entre 18 y 75 años provenientes de 160 hospitales en 34 países diferentes. Los pacientes elegibles tuvieron un primer evento clínico desmielinizante en un período de 75 días antes del screening, por lo menos dos lesiones clínicamente silentes de por lo menos $3 \mathrm{~mm}$ en una secuencia T2 de resonancia magnética, y un puntaje de escala EDSS de cinco o menos. 616 pacientes en total recibieron tratamiento, siendo este placebo o cladribina, esta última con dos dosificaciones distintas: $3.5 \mathrm{mg} / \mathrm{kg}$ y $5.25 \mathrm{mg} / \mathrm{kg}^{19}{ }^{20}$.

Al concluir el ensayo, la cladribina se había asociado con una reducción en el riesgo en el tiempo de conversión a una esclerosis múltiple clínicamente definida. Eventos adversos se documentaron en $81 \%$ y $82 \%$ de los grupos en tratamiento con cladribina, siendo mayor en el grupo con una dosis de $5.25 \mathrm{mg} / \mathrm{kg}$, contra tan solo $79 \%$ del grupo placebo. Los autores no encontraron un incremento en el riesgo de efectos adversos con el tratamiento activo contra placebo más allá de la linfopenia. Esta linfopenia fue sin embargo severa en 14 pacientes de la muestra total. Este estudio comparte muchas similitudes en su diseño con el CLARITY debido a que fue publicado después, y basa mucha de sus hipótesis en los hallazgos documentados por el estudio en cuestión 1920 .
El ensayo CLARITY fue un estudio clínico aleatorizado de una duración de 96 semanas, fase III de doble ciego, diseñado para valorar la efectividad en el tratamiento de la esclerosis múltiple con tabletas orales de la cladribina. El estudio tomó un total de 1326 pacientes, randomizados en una relación 1:1:1 a dos regímenes cortos de ingesta de cladribina $(3.5$ y $5.23 \mathrm{mg} / \mathrm{kg}$ de dosis acumulativa por el lapso total del ensayo en los dos grupos usando fármaco) o placebo. Periódicamente se monitorizaba eventos adversos, examen neurológico y físico de rutina, así como pruebas de laboratorio frecuentes para así valorar la seguridad de la terapéutica. De los pacientes randomizados, $88.6 \%$ completaron el tratamiento, mientras que $86.3 \%$ hicieron lo mismo con el placebo. El estudio encontró una reducción significativa de más de entre un $54-58 \%$ en la tasa anual de recaídas para los brazos activos del estudio al comparar contra placebo. También hubo resultados positivos en cuanto al el tiempo para la presentación de la primera recaída después de aplicado el tratamiento y la reducción de carga lesional en resonancia magnética y el tiempo para la presentación de la primera recaída después de aplicado el tratamiento. Referente a esto último se obtuvo que la proporción de pacientes sin lesiones activas al final del estudio en $\mathrm{T} 1 \mathrm{y}$ T2 fueron de 86.8 y $61.7,91.0-62.5$ respectivamente, esto en los grupos con las dos dosis diferentes de cladribina oral. El grupo de placebo mostró 48.3 y $28.4 \%$ respectivamente ${ }^{2122}$

Este estudio objetivizó además una mayor proporción de pacientes en estado libre de actividad de la enfermedad en el brazo de tratamiento con cladribina, definida esta condición como no volver a presentar un episodio de recaída, no empeoramiento sostenido en la escala de EDSS y no lesiones activas en resonancia magnética, valorándose tanto secuencias $\mathrm{T} 1$ y T2. El número total de días de hospitalización a lo largo de 96 semanas fue menor en los grupos recibiendo cladribina. A su vez, el número de visitas a los servicios de emergencia fue menor comparado contra el grupo de placebo, además del número promedio de visitas a clínicas. El tratamiento con cladribina también se reflejo en una reducción del número promedio de días de ausencia laboral. ${ }^{9}$ 23.

El CLARITY encontró como efectos adversos más común la linfopenia, lo cual era anticipado 
por el mismo mecanismo de acción de la cladribina. La incidencia de infecciones fue de $48.3 \%$ contra $45.2 \%$ entre los grupos de cladribina y placebo respectivamente. La infección con herpes zoster se desarrollo en $2.3 \%$ de los pacientes tratados con el quimioterapéutico. 9 pacientes tuvieron eventos relacionados a leiomiomas uterinos en el grupo de la cladribina contra tan solo uno en el grupo de placebo. 3 casos aislados de malignidad fueron reportados en el grupo de tratamiento con cladribina, un cuarto se documentó durante la vigilancia post-estudio. Un carcinoma cervical pre-maligno in situ también fue documentado $^{21}$

A pesar de los datos de eficacia presentados por este estudio, la European Medicines Agency (EMA), observando todos los efectos adversos citados, documentados en los estudios clínicos, se rehusó a aprobar el tratamiento con la cladribina para la esclerosis múltiple. Esto principalmente por el mayor riesgo de malignidades y de infecciones oportunistas, efectos adversos inherentes al mecanismo de acción. Otro punto de preocupación fue la posible reactivación de infecciones latentes tanto bacterianas como virales. Aunque la información fue insuficiente para establecer una clara relación causal entre el tratamiento con cladribina y el riesgo de malignidad, los análisis de ensayos de este fármaco sugerían fuertemente un potencial carcinogénico. Todo lo anterior llevó a que la casa farmacéutica recibiera retroalimentación negativa sobre este medicamento de parte de la FDA y la EMA, diciendo cesar en sus intentos de obtener una licencia de aprobación mundial para oficializar el uso de cladribina como tratamiento de esclerosis múltiple ${ }^{8924}$.

Desde ese punto sin embargo, la evidencia ha dado avances importantes referentes a las dudas planteadas sobre la seguridad y efectos adversos del medicamento. Muchos de estas novedades sobre el rol de la cladribina en el tratamiento se han determinado mediante análisis de la población delimitada por el mismo estudio CLARITY original.

Dentro de las primeras publicaciones claves en comenzar a cambiar el paradigma sobre la cladribina fue la realizada por Pakpoor et al, los cuales diseñaron un metaanálisis de ensayos fase III sobre diferentes drogas aprobadas para el tratamiento de esclerosis múltiple en su forma de brote-remisión, así como la inclusión del estudio
CLARITY. Las tasas de cáncer fueron comparadas utilizando la prueba exacta de Fisher. Al comparar los ensayos para drogas como cladribina, dimetilfumarato, fingolimod, teriflunamida, natalizumab, alemtuzumab y acetato de glatiramer, se concluyó que la tasa de cáncer en el grupo de tratamiento del ensayo CLARITY no fue mayor al compararse con los grupos de tratamientos para otro fármaco $(0.34 \%$ contra $0.6 \%)$. Este estudio concluyó que a pesar de que había previamente contribuido a la negación de la licencia en Europa e influenciado la postura de la FDA, el riesgo de cáncer con las dosis utilizadas en el CLARITY y en el ORACLE MS no se asociaba a un riesgo mayor de cáncer. El estudio además indica que la corta latencia entre iniciación del tratamiento y el diagnóstico de cáncer en el CLARITY de tan solo 18 meses causa una relación causal menos probable. En estas comparaciones entre fármacos es importante considerar que la cladribina en sus estudios fue comparada contra un grupo placebo, mientras que para muchas de las otras drogas, como el alemtuzumab, se utilizó un segundo grupo con comparador activo. No es posible concluir sin embargo, si la comparación con un grupo placebo podría exagerar el exceso de riesgo relativo ${ }^{25}$.

Otras conclusiones importantes fueron presentadas en el Consorcio de Centros de Esclerosis Múltiple (CMSC) de 2017 relativas a un análisis post hoc de 867 pacientes tomados de 1184 personas la población original del estudio CLARITY, el cual fue llamado CLARITY EXT, diseñado para comparar la seguridad y eficacia de 2 años adicional de cladribina contra no recibir tratamiento adicional. Este estudio post hoc tomó pacientes que habían placebo en el estudio original y se les asignó tratamiento con $3.5 \mathrm{mg} / \mathrm{kg}$, mientras que aquellos que habían recibido alguna dosis de cladribina en el CLARITY fueron rerandomizados asignados 2:1 a tabletas de cladribina $3.5 \mathrm{mg} / \mathrm{kg}$ o placebo, resultando en un total de cinco grupos. Los análisis demostraron que $73 \%$ a $90 \%$ de los pacientes que recibieron cladribina en los dos estudios permanecieron libres de nuevas relaciones en la secuencia T1, y $75 \%$ a $81 \%$ permanecieron libres de recaídas. Al compararse los diversos grupos de tratamiento, no hubo diferencia estadísticamente significativa en el porcentaje de pacientes libres de recaídas o en la proporción sin progresión confirmada a las 12 horas. En el análisis exploratorio sin embargo, la 
proporción de pacientes sin nuevas lesiones captantes de gadolinio en T1 fue significativamente menor en los grupos que recibieron placebo en el CLARITY EXT. La tasa anual de recaídas total fue de $0.13^{26}$

Referente al perfil de seguridad, los eventos adversos más comúnmente encontrados en pacientes tratados con cladribina fueron linfopenia, variando la incidencia en cada grupo de tratamiento. Esta fue mayor en el grupo que en el CLARITY habían recibido $5.23 \mathrm{mg} / \mathrm{kg}$ y en el análisis post hoc recibieron $3.5 \mathrm{mg} / \mathrm{kg}$; así mismo fue la aparición de linfopenia fue menor en el grupo que recibieron placebo en el CLARITY EXT posterior a haber sido tratados en el estudio original con $3.5 \mathrm{mg} / \mathrm{kg}$. La incidencia de infecciones fue bastante consistente entre los grupos, manejándose en un intervalo de $45.1 \%$ y $49 \%$. En un análisis integrado de seguridad tomando en cuenta los ensayos CLARITY, CLARITY y CLARITY EXT entre otros, la incidencia ajustada de eventos adversos por 100 pacientes-año para neoplasias fue 1.14 en los pacientes utilizando $3.5 \mathrm{mg} / \mathrm{kg}$ de cladribina y 1.01 en el grupo placebo. ${ }^{26}$

Otras publicaciones subsecuentes referentes al tratamiento con cladribina basadas en análisis posteriores del estudio CLARITY han demostrado beneficios en otros aspectos. Análisis del dataset del CLARITY sugiere que además de la eficacia clínica ya discutida, el tratamiento con el quimioterapéutico conlleva una mejora en la calidad de vida a lo largo de 96 semanas, objetivándose este parámetro con una correlación de rango de Spearman entre puntajes de Health-relatedquality of life (QuOL) y EDSS. Respuestas a los cuestionarios EQ-5D y MSQOL-53 fueron comparados entre los grupos de tratamiento y control El tratamiento con tabletas de cladribina siguiendo las pautas del estudio CLARITY se asoció además a una atrofia cerebral significativamente reducida 2728 .

Con esta información, en el año 2016 la casa farmacéutica anunció su reaplicación para una aprobación de la cladribina en el esquema terapéutico para la esclerosis múltiple en su forma brote-remisión. En el 2017 al analizar la evidencia presentada y comentada en este artículo, la EMA recomendó la aprobación de la cladribina, y aconsejó que el tratamiento fuera iniciado y supervisado por médicos experimentados en el abordaje de la esclerosis múltiple. Actualmente, esta soli- citud está siendo revisada por el NICE y SMC, los cuales dictaran su postura sobre la aprobación de este medicamento en mercados europeos. Se espera un comunicado oficial a inicios del 2018. La FDA no ha dictado postura al respecto. Serán importantes además los resultados del ensayo PREMIERE, un estudio de seguimiento a largo plazo a la información derivada de un estudio del mismo nombre. Este estudio de seguimiento consistirá en más de 10000 pacientes años de exposición en total, con el seguimiento en algunos pacientes excediendo los 8 años desde la compleción 2930313233

\section{CONCLUSIONES}

A pesar de haber sido apartado de las opciones terapéuticas para abordar la esclerosis múltiple en su forma brote-remisión debido a dudas sobre su seguridad referente a infecciones y mayor incidencia de neoplasias, principalmente malignas, la última evidencia apunta a un perfil de seguridad aceptable para la cladribina. A nivel mundial la tendencia es la incorporación de la cladribina a los diferentes esquemas de tratamiento, pudiendo tener su inclusión tan pronto como en el 2018 en los mercados europeos según el pronunciamiento de la NICE, pero contando ya con recomendación de la EMA.

En Costa Rica y como se desprende la Guía Nacional para el Tratamiento de Esclerosis Múltiple, nuestras decisiones referentes a cuales fármacos utilizar e incorporar en la terapéutica van más acorde a lo indicado por guías canadienses, latinoamericanas y principalmente las licencias de aprobación brindadas por la FDA, la cual todavía no se ha pronunciado referente a esta molécula. Sin embargo, conforme más y más entes reconozcan la evidencia de la cladribina arrojada en los últimos 6 años, y principalmente si la cladribina es completamente aprobada para su uso en países europeos, se tendrá que considerar la adición de esta droga oral a los tratamientos disponibles en nuestro país. Actualmente, la evidencia apunta hacia esta dirección.

\section{BIBLIOGRAFÍA}

1. Valverde A, Vargas R, Parajeles, A et al. Guía Nacional para el Tratamiento de 
la Esclerosis Múltiple. Neuroeje, 2015; 28 (2): 69-120.

2. Daroff R, Jankovic J, Mazziotta J et al. Bradley's Neurology in Clinical Practice. Seventh Edition. Chapter 80: Multiple Sclerosis and Other Inflammatory Demyelinating Diseases of the Central Nervous System. Elsevier. 2016; 1159-1186.

3. Hauser L, Stephenson A. Harrison's Neurology in Clinical Medicine. $4^{\text {th }}$ Edition. Chapter 45: Multiple Sclerosis and Other Demyelinating Diseases. McGrawHill Education. 2017;513-532.

4. Rooper A, Samuels M, Klein J. Adams and Victor's Principles of Neurology. Chapter 36: Multiple Sclerosis and Other Inflammatory Demyelinating Diseases. McGraw-Hill education, 2014;915-945.

5. Montalban X, Hauser S, Kappos L. Ocrelizumab versus Placebo in Primary Progressive Multiple Sclerosis. N Engl J Med 2017; 376(3): 209-220.

6. Roche. Media ReleaseFDA approves Roche's OCREVUS ${ }^{\mathrm{TM}}$ (ocrelizumab) for relapsing and primary progressive forms of multiple sclerosis. Obtenido de: http://www.roche.com/media/store/releases/med-cor-2017-03-29.htm

7. FDA. FDA Approves new drug to treat multiple sclerosis. Obtenido de https://www.fda.gov/newsevents/newsroom/pressannouncements/ucm549325. $\underline{\mathrm{htm}}$

8. Kim W, Edler M, Kim S et al. Oral Disease-Modifying Therapies for Multiple Sclerosis. J ClinNeurol 2015 Jan; 11(1): 9-19.

9. Paul F. Cladribine in multiple sclerosis: pitfalls in a new treatment landscape. Expert Opinion on Pharmacotherapy 2013; 14(1).
10. Gonzalez A. Esclerosis Múltiple afecta a tres mujeres por cada hombre. 2015. Obtenido de: http://www.revistaperfil.com/salud/Esclerosis-multiple-afectamujeres-hombre 0 743925601.html

11. Solis M. CCSS mantiene bajo tratamiento a 264 pacientes con Esclerosis Múltiple. 2013. Obtenido de: https://www.ccss.sa.cr/noticia?ccssmantiene-bajo-tratamiento-a-264-pacientes-con-esclerosis-multiple

12. Singh V, Prajeeth C, Gudi V et al. 2Chlorodeoxyadenosine (cladibrine) induces apoptosis in human monocytederived dendritic cells. ClinExpImmunol2013 Aug; 173(2): 288-297.

13. Alvarez $\mathrm{C}$, Adams A, Mathews $\mathrm{J}$ et al. Cladribine to treat disease exacerbation after fingolimod discontinuation in progressive multiple sclerosis. Ann ClinTransl Neurol. 2017 Jul; 4(7): 506511

14. Mitozek S, Tabarkiewiz J, Wilczynska $\mathrm{B}$ et al. Impact of cladribine therapy on changes in circulating dendritic cell subsets, $T$ cells and $B$ cells in patients with multiple sclerosis. $\mathrm{J}$ Neurol Sci. 2013 Sep 15;332(1-2):35-40

15. Laugel B, Borlat F, Galibert L et al. Cladribine inhibits cytokine secretion by $T$ cells independently of deoxycytidine kinasaactivity. Neuroimmunol. 2011 Dec 15;240-241

16. Baker D, Herrod S, Alvarez C et al. Both cladribine and alemtuzumab may effect MS via B-cell depletion. NeurolNeuroimmunolNeuroinflamm. $2017 \mathrm{Jul}$; 4(4): e360.

17. Savic R, Novakovic A, Ekblom M et al. Population Pharmacokinetics of Cladribine in Patients with Multiple Sclerosis. ClinPharmacokinet. 2017. 
18. Rice GP, Filippi M, Comi G. Cladribine and progressive MS: clinical and MRI outcomes of a multicenter controlled trial. Cladribine MRI Study Group. Neurology 2000; 54: 1145-1155

19. Leist T, Comi G, Cree B. Effect of oral cladribine on time to conversion to clinically definite multiple sclerosis in patients with a first demyelinating event (ORACLE MS): a phase 3 randomised trial.Lancet Neurol. 2014 Mar;13(3):257-67.

20. EMD Serono. Oral Cladribine in Early Multiple Sclerosis (MS) (ORACLE MS). 2013. Obtenido de: https://clinicaltrials.gov/ct2/show/NCT0 0725985

21. Cook S, Vermersch P, Comi G et al. Safety and tolerability of cladribine tablets in multiple sclerosis: the CLARITY (CLAdRibine Tablets treating multiple sclerosis orally) study. MultScler. 2011 May;17(5):578-93

22. Comi G, Cook S, Giovannoni G. MRI outcomes with cladribine tablets from multiple sclerosis in the CLARITY study. J Neurol. 2013 Apr;260(4):113646.

23. Ali S, Paracha N, Cook $\mathrm{S}$ et al. Reduction in healthcare and societal resource utilization associated with cladribine tablets in patients with relapsing-remitting multiple sclerosis: analysis of economic data from the CLARITY Study. Clin Drug Investig. 2012 Jan 1;32(1):15-27.

24. Comi G, Hartung H, Kurukulasuriya N et al. Cladribine tablets for the treatment of relapsing-remitting multiple sclerosis. Expert OpinPharmacother. 2013 Jan;14(1):12336
25. Pakpoor J, Disanto G, Altmann D et al. No evidence for higher risk of cancer in patients with multiple sclerosis taking cladribine. NeurolNeuroimmunolNeuroi nflamm. 2015 Dec; 2(6): e158.

26. Helwick V. Cladribine back in the Running for Multiple Sclerosis. Medscape 2017. Obtenido de: http://www.medscape.com/viewarticle/8 80990\#vp 2.

27. Afolabi D, Albor C, Zalewski L et al. Positive impact of cladribine on quality of life in people with relapsing multiple sclerosis. MultScler. 2017 Aug $1: 1352458517726380$

28. De Stefano N, Giorgio A, Battaglini M et al. Reduced brain atrophy rates are associated with lower risk of disability progression in patients with relapsing multiple sclerosis treated with cladribine tablets. MultScler. 2017 Jan 1:1352458517690269

29. MS Society. Cladribine. 2017. Obtenido de: https://www.mssociety.org.uk/cladribine

30. Brooks M. CHMP Recommends Cladribine (Mavenclad) for Multiple Sclerosis. 2017. Obtenido de: http://www.medscape.com/viewarticle/8 $\underline{82043}$

31. Mstrust. Cladribine (Mavenclad). 2017. Obtenido de: https://www.mstrust.org.uk/a-z/cladribine-mavenclad

32. Merck. Cladribinetablets receives positive CHMP Opinion for Treatment of Relapsing Forms of Multiple Sclerosis.2017. Obtenido de: https://www.merckgroup.com/content/d am/web/corporate/non-images/press-releases/2017/jun/en/Mavenclad-CHMP23-06-2017-EN.pdf 
33. EMD Serono. Prospective Observational Long-Term Safety Registry of Multiple Sclerosis Patients who Have Participated in Cladribine Clinical Trials (PREMIERE).2017. Obtenido de: https://clinicaltrials.gov/ct2/show/NCT0 $\underline{1013350}$

\section{CONFLICTO DE INTERÉS Y/O AGRADECIMIENTOS}

Los autores declaran que no existió ningún conflicto de interés en el presente reporte. 